\title{
Les spermatozoïdes immobiles frais ou décongelés en fécondation assistée
}

Yigal Soffer1, Deborah Strassburger2“ , Shevach Friedler2 , Sarita Kaufman1“ , Anna Umanski1“ Arié Raziel2", Raphaël Ron-El 2

1Centre d'infertilité masculine, \& 2Unité de FIV Centre médical Assaf Harofé" Zerifin Faculté de médecine Sackler, Université de Tel-Aviv, Israël.

\section{RÉSUMÉ}

Contrairement au compte ou à la morphologie des spermatozoïdes, l'absence totale de mobilité du sperme peut abaisser les taux de fécondations et de grossesses en micro injection. Dans trois cas d'anomalies du squelette flagellaire (absence des bras de dynéine) l'éjaculat ne contenait que des spermatozoïdes immobiles. Après éjaculations répétées, on a pu souvent découvrir dans ces cas un taux plus élevé de spermatozoïdes viables et même quelques spermatozoïdes mobiles. Par ailleurs, dans les azoospermies sécrétoires, la recherche in extremis ne permet parfois de trouver que peu de spermatozoïdes, pour la plupart immobiles avec quelques rares spermatozoïdes mobiles, insuffisants pour tous les ovocytes. Le troisième groupe de sperme immobile en fécondation assistée, iatrogène, est consécutif à la cryoconservation de spermatozoïdes surnuméraires non éjaculés, fragments testiculaires, aspirats épididymaires, faite pour éviter des prélèvements chirurgicaux répétés. Après décongélation, on ne retrouve parfois que de rares spermatozoïdes mobiles et l'on est alors forcé d'injecter des ovocytes avec du sperme immobile. Ce travail a pour objet d'évaluer les résultats de la micro injection avec du sperme immobile dans les trois groupes susmentionnés: 1- sperme immobile avec anomalies flagellaires, 2spermatozoïdes immobiles découverts après recherche " in extremis ", 3- sperme décongelé immobile. Les résultats de la micro injection montrent que le pouvoir fécondant en
ICSI des spermatozoïdes immobiles, frais ou décongelés n'est pas très différent de celui des spermes mobiles de même origine. Plus encore, dans le groupe des anomalies flagellaires avec nécrozoospermie, des éjaculations répétées permettent d'améliorer le taux de viabilité des spermatozoïdes. La mise en évidence chez un couple d'un sperme totalement immobile, frais ou décongelé, ne devrait donc pas nous amener à renoncer à la micro injection. Des grossesses ont été ainsi obtenues avec le sperme du mari.

Mots Clés: Spermatozoïdes immobiles, nécrozoospermie, anomalies flagellaires, azoospermies, recherche de spermatozoïdes, prélèvement chirurgical de spermatozoïdes; cryo préservation; fécondation assistée; taux de Fécondations; taux de grossesses.

Correspondance:

Yigal Soffer M.D. Male Infertility Clinic, Assaf Harofé

Medical Center, Zerifin 70300, Israel

Tel: (972) 8-977 9690 Fax: (972) 8-977 9691

E-mail: ysoffer@asaf.health.gov.il 


\section{INTRODUCTION}

L'injection intracytoplasmique (ICSI) permet d'obtenir un taux élevé de fécondations et de grossesses [21,31,32] chez des couples souffrant d'infécondité masculine extrêmement grave. Le compte et la morphologie des spermatozoïdes n'interfèrent pratiquement pas avec les résultats de l'ICSI. Par contre. l'absence totale de mobilité du sperme porte une sérieuse atteinte aux taux de fécondations et de grossesses [15, 16, 18,33]. Lorsqu'il s'agit aussi d'une nécrozoospermie, les résultats sont encore plus désastreux.

Dans certaines de ces nécrozoospermies, on trouve des anomalies flagellaires d'origine génétique, décelables en microscopie électronique de transmission [38]. Mais le plus souvent il s'agit d'éjaculats considérés " azoospermiques ", où la recherche in extremis systématique de spermatozoïdes [24] permet dans un nombre non négligeables de cycles, près de $30 \%$, de découvrir des spermatozoïdes mobiles en nombre suffisant pour pratiquer la micro injection des ovocytes aspirés. Mais il arrive aussi que le nombre de spermatozoïdes mobiles disponibles soit fort limité. Lorsque les ovocytes sont plus nombreux que les spermatozoïdes mobiles, l'on se trouve devant le dilemme d'injecter des spermatozoïdes immobiles ou de prélever des spermatozoïdes testiculaires. A l'Hôpital Assaf Harofé, pour éviter d'éventuels prélèvements chirurgicaux de spermatozoïdes dans des cycles ultérieurs de tous les cas difficiles, les spermatozoïdes viables surnuméraires sont congelés après micro injection $[7,8]$. Là encore, après décongélation, il n'est pas rare de se trouver confronté au problème de spermatozoïdes décongelés immobiles.

Cette revue de la littérature est tout naturellement centrée sur notre expérience dans les cycles difficiles de micro injection avec spermatozoïdes immobiles. L'accent est mis sur la conduite tenue et les résultats qu'elle a donnés.

\section{SPERMATOZOÏDES IMMOBILES AVEC ANOMALIES FLAGELLAIRES}

Dans ces cas d'asthénozoospermie totale, d'origine génétique, il y a généralement une anam- nèse familiale de syndrome de cils immobiles $[1,5]$. Le diagnostic est généralement confirmé à la microscopie électronique [5,37] qui démontre l'absence de bras de dynéine [1]. Il y a également une nécrozoospermie importante $[12,13,33]$. Cette nécrozoospermie serait due à la stagnation des spermatozoïdes dans les voies génitales masculines, les spermatozoïdes perdant leur vitalité durant ce séjour prolongé dans le rete testis, la queue de l'épididyme et le canal déférent [30]. Il se pourrait également que, durant cette rétention prolongée, un nombre accru de spermatozoïdes subirait une oxydation des radicaux sulfhydriques de la chromatine spermatique [14], la rendant inapte à se décondenser [28] et à féconder. Avant l'ère de l'ICSI, des fécondations et des grossesses ont été rapportées dans les dyskinésies flagellaires avec absence de bras de dynéine, mais il n'y avait pas une absence totale de mobilité [37]. Nijs et coll.[19] en 1996 ont décrit un cas de Kartagener avec spermatozoïdes totalement immobiles traité par l'insémination sub-zonale des ovocytes, avec fécondation de 12 ovocytes et une grossesse.

Nijs et coll. [19] en1996, ont rapporté également un taux de fécondation de $53 \%$ après ICSI avec du sperme totalement immobile. 36 embryons avaient été transférés chez 12 patientes. Aucune grossesse ne fut obtenue. Cependant dans ces cas les bras extérieurs de dynéine n'étaient pas absents. Nijs et al. [19] en 1996 ont également rapporté un cas de Kartagener sans aucune fécondation après ICSI de quatre ovocytes. Papadimas et coll. [22] ont rapporté en 1997 un cas avec un taux de fécondations de $33 \%$ sans grossesse. Mais le taux de viabilité était de $72 \%$. Pour Kahraman et coll. [12] en1996, on n'obtient avec les spermatozoïdes éjaculés qu'un très faible taux de grossesses en micro injection et pas de grossesses évolutives du tout. Aussi, Kahraman et coll., [13] ainsi que Silber et coll.[27] préconisent-ils le prélèvement de spermatozoïdes testiculaires dans les cas de spermatozoïdes totalement immobiles dans l'éjaculat. Dans sa série de cas, Kahraman et coll., [13] rapportent en1997 l'accouchement d'un enfant bien portant et cinq grossesses évolutives obtenus par ICSI avec du sperme testiculaire néanmoins totalement immobile mais avec une vitalité 
satisfaisante de $79 \%$, alors qu'elle n'était que de $17 \%$ dans l'éjaculat.

Ce n'est que récemment que des grossesses avec sperme éjaculé totalement immobile ont été rapportés en fécondation assistée. Dans deux cas avec une très bonne viabilité, $90 \%$, des spermatozoïdes, von Zumbusch, [35] et coll. ont rapporté en 1998 deux grossesses, dont une gémellaire, au premier cycle de traitement.

Dans trois cas de spermatozoïdes totalement immobiles et investigués dans notre centre d'infertilité masculine avec l'aide d'un laboratoire de microscopie électronique, une série de cycles de micro injections avec le sperme éjaculé a été rapportée par Ron-El et coll. en1998 [25]. Cette série de cas présente un certain intérêt à cause de la sévérité de la nécrozoospermie associée et de l'investigation de la viabilité des spermatozoïdes faite après abstinence standard et à la suite d'éjaculations répétées. Aux premiers cycles de traitements, l'épreuve à l'éosine-Y $[3,36]$ après abstinence standard montrait dans ces trois cas un taux de vitalité de $41 \%$ en moyenne. Après microinjection, un seul ovocyte sur un total de 39 , pour les trois cas, avait été fécondé et transféré. Il n'y a pas eu de grossesse. D'autres épreuves de vitalité $[10,11]$ peuvent être pratiquées mais elles sont plus délicates à utiliser. Dans les cycles suivants, des éjaculations plus fréquentes avaient été demandées dans la semaine précédant l'aspiration des ovocytes et deux à trois éjaculats consécutifs, ont été obtenus le jour de la ponction ovocytaire. L'épreuve à l'éosine montrait dans les derniers éjaculats une vitalité accrue [71\% en moyenne] des sper- matozoïdes, permettant un taux sensiblement plus élevé de fécondations [Tableau 1] ainsi qu'une grossesse évolutive de jumeaux bien portants.

Certes, l'accroissement du taux de fécondations et l'obtention d'une grossesse gémellaire après éjaculations répétées sont surprenant. Il n'en reste pas moins que la simple demande d'éjaculats répétés peut permettre, sans prélèvement testiculaire, d'obtenir dans ces nécrozoospermies des résultats fort satisfaisants en fécondation assistée. Il faudrait pourtant s'assurer du risque héréditaire dans la progénie de ces couples, d'autant plus que des anomalies congénitales aient été décrites dans des cas familiaux de Kartagener [2, 5]. Il est encore trop tôt pour savoir si les anomalies décelées dans les spermatozoïdes du père ont été transmis à la descendance.

\section{SPERME ÉJACULÉ FRAIS, RECHER- CHÉ IN EXTREMIS DANS LES AZOO- SPERMIES SÉCRÉTOIRES.}

Lorsqu'on peut de nos jours, avec quelques spermatozoïdes épars obtenir des fécondations et des grossesses, le concept de l'azoospermie, défini simplement par l'absence de spermatozoïdes dans le liquide séminal mérite une analyse plus fouillée. Tournaye et col., [29] en 1995 distinguent l' azoospermie "absolue" de l' azoospermie "virtuelle". Dans l'azoospermie "absolue" les spermatozoïdes sont absents dans tous les éjaculats même après centrifugation. Dans l' azoospermie "virtuelle" les spermatozoïdes, néanmoins absents le jour de la ponction des ovocytes, pouvaient être présents dans l'éjaculat, dans un ou plusieurs examens précédents,.

Tableau 1: Fécondations normales, clivage d'embryons et embryons transférés au premier cycle et durant les cycles répétés de micro-injection avec sperme totalement immobile et anomalies flagellaires [25].

\begin{tabular}{lllll}
\hline $\begin{array}{l}\text { Ovocytes } \\
\text { injectés }\end{array}$ & $\begin{array}{c}\text { Fécondations } \\
\text { à } 2 \mathrm{n}\end{array}$ & $\begin{array}{l}\text { Embryons } \\
\text { clivés }\end{array}$ & $\begin{array}{l}\text { Cycles avec } \\
\text { transfert }\end{array}$ & $\begin{array}{l}\text { Embryons/ } \\
\text { transfert }\end{array}$ \\
$\begin{array}{c}\text { 1ers cycles: } \\
39\end{array}$ & $1 / 39^{*}$ & $1 \mathrm{a}$ & $1 / 3$ & 1 \\
$\begin{array}{c}\text { Cycles répétés: } \\
85\end{array}$ & $41 / 85^{*}$ & $35^{*}$ & $6 / 6$ & $3.2 \pm 0.9$ \\
\hline
\end{tabular}

$\left.{ }^{*} \mathrm{p}<0.0001\right]$ 
Dans quatre cas sur 15 "d'azoospermie absolue " aucun spermatozoïde n'a pu être trouvé dans la biopsie testiculaire. [29]. Dans un travail plus récent du même groupe [30], dans huit cas (28\%) d' "azoospermie absolue" sur 29 , aucun spermatozoïde n'a été trouvé dans le prélèvement testiculaire. Ils ont par contre été découverts dans les prélèvements testiculaires de 25 cas “ d'azoospermie virtuelle."

Dans 51 cas longuement investigués d'azoospermie considérée "absolue", à la suite de plusieurs recherches négatives de spermatozoïdes, Ron-El et coll. [24], 1997, ont pratiqué le jour de la ponction des ovocytes une recherche in extremis de spermatozoïdes dans les éjaculats. Dans 31 de ces cas, [65\%] cette recherche a été négative et un prélèvement testiculaire [6] a dû être pratiqué, mais dans 17 cas (35\%), des spermatozoïdes viables ont pu être trouvés. Dans 11 de ces cas, tous les ovocytes, 164 au total, avaient été injectés avec des spermatozoïdes mobiles. Dans 5 cas, les spermatozoïdes mobiles étaient moins nombreux que les ovocytes aspirés. Trente sept ovocytes sur $64(58 \%)$ avaient pu être injectés avec des spermatozoïdes mobiles tandis que 27 ovocytes $(42 \%)$ ne recevaient que des spermatozoïdes immobiles. Dans un cas, tous les 14 ovocytes avaient été injectés avec des spermatozoïdes immobiles. Pour replacer ces chiffres dans le contexte général des azoospermies dites " absolues, " on peut trouver des spermatozoïdes dans $35 \%$ de ces cas, mais dans $22 \%$ seulement, il est possible d'injecter tous les ovocytes ponctionnés avec des spermatozoïdes mobiles. Dans $10 \%$ de cas supplémentaires, il y a suffisamment de spermatozoïdes mobiles pour plus de la moitié (58\%) des ovocytes. Au total, 201 (82.7\%) ovocytes avaient été injectés avec des spermatozoïdes mobiles et $42(17.3 \%)$ ovocytes avaient reçu des spermatozoïdes immobiles. Le Tableau 2 compare les résultats de la micro injection dans ces deux groupes.

Aucune différence significative n'avait été trouvé dans le taux de fécondation lorsque les spermatozoïdes était mobiles (39\%) ou immobiles $(26 \%)$ mais toutes les quatre grossesses avaient été obtenues avec des spermatozoïdes mobiles. Pour Vandervorst et coll. [33], 1997, le taux de fécondation avec sperme éjaculé totalement immobile est sensiblement plus faible qu'avec des spermes mobiles. Ces auteurs n'ont eu aucune grossesse évolutive avec des spermes éjaculés totalement immobiles.

\section{AVEC DES SPERMATOZOÏDES DÉCONGELÉS IMMOBILES}

Dans les azoospermies non-obstructives, qui sont certainement les formes cliniques les plus graves de l'infécondité masculine le nombre de spermatozoïdes dans les testicules est souvent très bas. La micro injection cytoplasmique permet néanmoins de cryopréserver des spermatozoïdes surnuméraires après toute biopsie testiculaire, qu'elle soit thérapeutique ou diagnos-

Tableau 2: Résultats de la micro injection avec sperme mobile et immobile après recherche in extremis de spermatozoüdes dans des éjaculats dits azoospermiques [24]. Aucune différence significative n'a été trouvée entre les deux groupes. Les pourcentages se réfèrent aux 1 ovocytes, aux oufs 2 fécondés normaux, et aux ${ }^{3}$ embryons obtenus.

\begin{tabular}{|c|c|c|}
\hline Oeufs et embryons & Sperme mobile & Sperme Immobile \\
\hline 1 - Ovocytes injectés & 201 & 42 \\
\hline 2 - Fécondations à $2 \mathrm{PN}$ & $78 \quad[39 \%]^{1}$ & $11[26 \%]^{1}$ \\
\hline 3 - Embryons, au total & $77[38 \%]^{1}[99 \%]^{2}$ & $9 \quad[21 \%]^{1} \quad[82 \%]^{2}$ \\
\hline - Excellents & $16[21 \%]^{3}$ & $1[11 \%]^{3}$ \\
\hline - Bons & $20 \quad[26 \%]^{3}$ & $4[44 \%]^{3}$ \\
\hline
\end{tabular}

$\mathrm{p}=$ n.s. pour tous 
tique $[4,9,16,1720]$. En effet certains couples préfêrent, par une biopsie préalable, avoir la certitude de disposer de spermatozoïdes testiculaires avant d'entrer en cycle de fécondation assistée. Les spermatozoïdes prélevés sont alors cryopréservés dans l'attente d'un cycle de micro-injection. Ce mode de traitement en deux temps semble également donner des résultats satisfaisant [20].

Il n y a pas de différence significative dans les résultats de la micro-injection entre les spermatozoïdes non éjaculés frais ou décongelés $[7,8,9.17,32]$ en ce qui concerne les taux de fécondation, de clivage, ou de nidation. Les grossesses évolutives seraient néanmoins moins nombreuses avec le sperme testiculaire congelé [4]. C'est également ce qui semble ressortir de nos séries $[7,8]$, mais elles ne sont pas encore assez grandes pour en tirer des conclusions définitives. Tous les auteurs s'accordent à penser qu'en permettant de réitérer des cycles thérapeutiques et de cumuler des grossesses, la cryopréservation de spermatozoïdes non éjaculés augmente notablement l'efficacité de la micro injection $[7,8]$ sans le recours à des prélèvements chirurgicaux répétés.

Néanmoins, aucune évaluation qualititative des dégâts cellulaires à l'échelon des spermatozoïdes testiculaires épars n'a été rapporté à notre connaissance. Comme beaucoup d'autres [32], nous congelons les spermatozoïdes testiculaires en suspension après simple dilacération du tissu germinatif à l'aide d'un protocole de congélation très simple à deux temps $[7,8]$. D'autres $[23,32]$ se servent d'un protocole à refroidissement lent contrôlé par ordinateur sans obtenir de meilleurs résultats. L'isolement des spermatozoïdes non éjaculés de bonne qualité avant la congélation a été suggéré [32]. Elle n'est praticable que dans les azoospermies obstructives ou lorsque les spermatozoïdes non-éjaculés sont abondants. Aussi, n'est-il pas étonnant d'obtenir après décongélation un très faible taux de spermatozoïdes mobiles. Il faut généralement pratiquer une recherche minutieuse pour trouver quelques spermatozoïdes mobiles et il n'est pas rare de n'en avoir pas assez pour tous les ovocytes. On doit alors injecter dans certains ovocytes des spermatozoïdes immobiles.
L'injection de spermatozoïdes prélevés par ponction épididymaire percutanée [PESA], cryopreservés et décongelés a été faite [26] dans 35 cycles. Dans deux de ces cycles après PESA, des spermatozoïdes décongelés immobiles ont été injectés.

Linjection de spermatozoïdes prélevés par biopsie testiculaire [TESE], cryopreservés et décongelés a été faite dans 19 cycles [26]. Dans sept de ces cycles après TESE, des spermatozoïdes décongelés immobiles ont été injectés. Dans ces cycles, les résultats de la micro injection de spermatozoïdes décongelés mobiles et immobiles ont été les suivants :

1. Dans deux cycles utilisant du sperme épididymaire cryopreservé après PESA et décongelé, il y avait18 ovocytes à injecter. Huit ovocytes avaient été injectés avec du sperme immobile. Un seul embryon s'est développé, tandis que dans les dix autres ovocytes, injectés avec du sperme mobile, quatre embryons étaient obtenus. Cette différence n'était pas significative. $14 \%$ des ovocytes injectés avec des spermatozoïdes immobiles n'avaient qu'un seul pronucléus, alors que $6 \%$ des ovocytes ayant reçu du sperme mobile étaient de ce type (différence non significative). La qualité des embryons, la morphologie et le nombre des blastomères étaient sensiblement les mêmes, indifféremment de la mobilité des spermatozoïdes injectés. Dans ces deux cycles, des embryons avaient pu être transférés mais il n'y a pas eu de grossesse.

2. Dans sept cycles utilisant du sperme testiculaire cryopréservé après TESE, il y avait114 ovocytes à injecter : Dans les 51 ovocytes ayant reçu du sperme immobile, le taux de fécondation était de $31 \%$ et celui du clivage $75 \%$. Quant aux 63 ovocytes ayant reçu du sperme mobile, les taux respectifs de fécondation et de clivage, $37 \%$ et $87 \%$, n'étaient pas sensiblement différents. Des embryons de qualité satisfaisante avaient été transférés dans tous ces cycles, indifféremment de la mobilité du sperme injecté. Quatre grossesses avaient été enregistrées, d'autant plus remarquables que trois d'entre elle étaient gémellaires à leur début, de sorte qu'en fait, sept sacs embryonnaires s'étaient développés. L'une des grossesses gémellaires donna naissance à deux 
enfants normaux. La seconde se réduisit spontanément à un seul foetus au cours de la grossesse et aboutit à la naissance d'un seul enfant bien portant. La troisième grossesse, gémellaire, et la quatrième grossesse, simple, finirent par des fausses couches. Ces résultats montrent avec certitude qu'au moins deux sacs embryonnaires, celui de la grossesse simple et celui de l'un des jumeaux avaient été obtenus avec des spermatozoïdes totalement immobiles. Néanmoins, ces deux sacs ne se développèrent pas au delà du premier trimestre.

Ainsi donc la micro injection de spermatozoïdes décongelés immobiles, faite lorsque l'on n'a pas d'autre choix, n'est pas une procédure vaine en termes de fécondations, clivages et nidations, de taux peu différents de ceux obtenus avec des spermatozoïdes mobiles. On ne peut cependant pas ignorer le léger avantage des chiffres en faveur des spermatozoïdes mobiles, surtout en ce qui concerne les grossesses avancées. Mais nos séries sont encore trop petites pour en tirer des conclusions.

Le pouvoir fécondant du sperme testiculaire cryopréservé et décongelé est vivement discuté. Verheyen et coll. [34] confirme en 1997 la baisse irréversible de mobilité de ces spermatozoïdes, leur vulnérabilité aux traitements in vitro et au Percoll en particulier. Dans les azoospermies obstructives, cette dégradation est généralement bien supportée (dans 29 cas sur 31) selon notre expérience à cause du grand nombre de spermatozoïdes que l'on peut aspirer de l'épididyme.

Il en est tout autrement dans les azoospermies sécrétoires où d'emblée, le petit nombre et la mauvaise qualité du sperme inhérents à l'insuffisance testiculaire ne permettent aucune récupération de mobilité par culture [16] et compromettent les chances de trouver des spermatozoïdes mobiles après décongélation. Nous n'en avons trouvé que 12 fois sur 19 [26]. Des grossesses avancées et des accouchements ont été rapportés avec du sperme testiculaire immobile frais [32] mais non après décongélation. Dans deux cas nous sommes sûrs d'avoir obtenu des grossesses cliniques avec des spermatozoïdes testiculaires décongelés immobiles, mais ces grossesses ne dépassèrent pas le premier trimestre. Il est important d'éviter autant que possible des prélèvements testiculaires et en dépit des doutes que suscitent nos résultats il faut continuer nos traitements avec du sperme cryopréservé et pour cela, améliorer nos techniques de cryopréservation, de décongélation et de traitement in vitro des spermes décongelés.

\section{CONCLUSION}

L'absence totale de spermatozoïdes mobiles pose un sérieux défi à la reproduction médicalement assistée surtout lorsque le nombre de spermatozoïdes disponibles est très faible et qu'il est difficile de juger de leur vitalité avant la micro-injection. Mais il s'avère que ce défi n'est pas insurmontable.

Dans les cas d'immobilité "totale" du sperme avec anomalies flagellaires, le problème essentiel est liée à une nécrozoospermie surajoutée, probablement en raison de l'effet délétère $\mathrm{du}$ séjour prolongé des spermatozoïdes dans le tractus génital mâle. Aussi, dans ces cas, sans aucune intervention sur les testicules, des éjaculations répétées nous ont permis d'obtenir des spermatozoïdes de meilleure vitalité capables de féconder en micro-injection et même de donner des grossesses.

Dans les azoospermies sécrétoires, il arrive qu'à la suite d'une recherche in extremis de spermatozoïdes dans l'éjaculat, faite pour éviter un prélèvement de sperme testiculaire, nous ne trouvons pas suffisamment de spermatozoïdes mobiles pour tous les ovocytes collectés et que nous soyons obligés d'injecter des ovocytes avec des spermatozoïdes immobiles. Nous n'avons pas relevé de grossesses avec ces ovocytes mais les taux de fécondations avec spermes mobiles et immobiles étaient similaires.

Quant aux spermatozoïdes décongelés immobiles, il semblerait que leur pouvoir fécondant ne soit pas très différent de celui des spermes décongelés mobiles. Il semblerait donc qu'en micro-injection, la mobilité et la viabilité des spermatozoïdes soient vraiment critiques avant et non après la congélation.

Ainsi donc, la mise en évidence chez un couple d'un sperme totalement immobile, frais ou décongelé, ne devrait donc pas nous amener à 
renoncer à la micro-injection. Le prélèvement testiculaire ou sa répétition ne sont également pas inéluctables, pas plus que le don du sperme. Le sperme immobile peut féconder et même, dans un nombre non négligeable de cas, donner des grossesses.

\section{REFERENCES}

1. AFZELIUS, B.A., ELIASSON, R., JOHNSEN, O. et coll.: Lack of dynein arms in immotile human spermatozoa. J. Cell Biol.,1975; 66: 225-8.

2. ALONSO, S. :Recherche de gene[s] implique[s] dans le situs inver-sus.Resultats preliminaires. Arch. Mal. Coeur Vaiss., 1991; 84: 633-7.

3. BLOM, E. [1950] A one min live-dead sperm stain by means of eosin-nigrosin. Fertil. Steril., 1, 176-7

4. DE CROO I, VAN DER ELST J, EVERAERT K, et coll.: Fertilization, pregnancy and embryo implantation rates after ICSI with fresh or frozen-thawed testi-cular spermatozoa. Hum Reprod 1998 Jul;13[7]:1893-7

5. ESCUDIER, E., ESCALIER, D., PINCHON, M.C. et coll. : Dissimilar expression of axonemal anomalies in respiratory cilia and sperm flagella in infertile men. Am. Rev. Respir. Dis.,1990 ; 142, 674-9.

6. FRIEDLER S, RAZIEL A, STRASSBURGER D. et coll.: Testicular sperm retrieval by percutaneous fine needle sperm aspiration [TEFNA] compared with testicular sperm extraction [TESE] by open biopsy in men with non-obstructive azoospermia. Human Reprod 1997, 12; 1488-93.

7. FRIEDLER S, RAZIEL A , SOFFER Y et coll.: Intracytoplasmic injection of fresh and cryopreserved testicular spermatozoa in patients with nonobstructive azoospermia - a comparative study; Fertil Steril 1997; 68: 892-7

8. FRIEDLER S, RAZIEL A, SOFFER Y, et coll., The outcome of intracytoplasmic injection of fresh and cryopreserved epididymal spermatozoa from pa-tients with obstructive azoospermia - a comparative study. Human Re-prod 1998, 13:1872-7

9. GIL-SALOM M, ROMERO J, MINGUEZ Y, et coll,,: Pregnancies after intracyto-plasmic sperm injection with cryopreserved testicular spermatozoa. Hum Reprod, 1996;11:1309-13

10. JASSIM, A., DRUDY, L. AND RAJAH, S.V : The use of GDA-J/F3 monoclonal antibody for the detection of dead spermatozoa [necrospermia] during semen analysis. Hum. Reprod., 1995 ; 10, 2301-4.

11. JEYENDRAN, R.S., VAN DER VEN, H.H., PEREZPALAEZ, M. et coll.: Development of an assay to assess the functional integrity of the human sperm mem-brane and its relationship to other semen characteristics. J. Reprod. Fer-til.,1984; $70: 219-23$.
12. KAHRAMAN, S., TASDEMIR, M., TASDEMIR, I. et coll.: Pregnancies achieved with testicular and ejaculated sperm in combination with intracytoplas-mic sperm injection in males with totally immotile or initially immotile spermatozoa in the ejaculate. Hum. Reprod.,1996; 11: 1343-6.

13. KAHRAMAN, A.Z. ISIK , K. VICDAN, S. OZGUR, O.D. Ozgun. A healthy birth af-ter intracytoplasmic sperm injection by using immotile testicular spermatozoa in a case with totally immotile ejaculated spermatozoa before and after Percoll gradients. Human Reproduction, 1997; 12: 292-3

14. KOSOWER N. S., KATAYOSE, H., YANAGIMACHI, R. : Thiol-disulfide status and acridine orange fluorescence of mammalian sperm nuclei. J. Androl., 1992,13: 342-8.

15. LIU, J., NAGY, Z., JORIS, H. et al.: Analysis of 76 total fertilization failure cycles out of 2732 intracytoplasmic sperm injection cycles. Hum. Reprod., 1995; 10: 2630-6.

16. LIU J, TSAI YL, KATZ E, et coll.: Outcome of in-vitro culture of fresh and frozen-thawed human testicular spermatozoa. Hum Reprod., 1997;12[8]:1667-72.

17. MADGAR I, HOURVITZ A, LEVRON J, et coll. : Outcome of in vitro fertilization and intracytoplasmic injection of epididymal and testicular sperm ex-tracted from patients with obstructive and nonobstructive azoospermia. Fertil Steril, 1998; 69:1080-4

18. NAGY, Z., LIU, J., JORIS, H. et coll,: The result of intracytoplasmic sperm injection is not related to any of the three basic sperm parameters. Hum. Reprod, 1995 ; 10: 1123-9.

19. NIJS, M., WANDERZWALMEN, P., VANDAMME, B. et al. : Fertilizing ability of immotile spermatozoa after intracytoplasmic sperm injection. Hum Re-prod., 1996; 11: 2180-5.

20. OATES RD, MULHALL J, BURGESS C, CUNNINGHAM D, CARSON R. : Fertilization and pregnancy using intentionally cryopreserved testicular tissue as the sperm source for intracytoplasmic sperm injection in 10 men with non-obstructive azoospermia., Hum Reprod 1997 ;12:734-9

21. PALERMO G., JORIS H., DEVROEY P., et coll.: Pregnancies after intracytoplas-mic sperm injection of sperm injection. Lancet, 1992; 340: 17-8.

22. PAPADIMAS J., TARLATZIS B.C., BILI H. et coll.: Therapeutic approach of im-motile cilia syndrome by intracytoplasmic sperm injection: a case report. Fertil. Steril., 1997; 67: 562-5.

23. PODSIADLY BT., WOOLKOTT RJ., STANGER JD. and Stevenson K. Case report: Pregnancy resulting from intracytoplasmic injection of cryopreserved spermatozoa recovered from testicular biopsy. Hum Reprod, 1996; 11: 1306-8.

24. RON-EL R., STRASSBURGER D., FRIEDLER S. et coll. : Extended sperm prepara-tion: an alternative to testicular sperm extraction in non-obstructive azoospermia. Hum. Reprod.,1997 ; 12, 1222-6. 
25. RON-EL R., STRASSBURGER D., FRIEDLER S. et coll.: Repetitive ejaculation be-fore intracytoplasmic sperm injection in patients with absolute immotile spermatozoa, Hum. Reprod, $1998 ; 13$ : 630-3.

26. RON-EL R, STRASSBURGER D, SOFFER Y, et coll. Fertilizing capability of frozen-thawed immotile sperm In: Hamamah $\mathrm{S}$ ed. Motility disorders... New York, Springer Verlag 1999: xxx-xx

27. SILBER S.J., VAN STEIRTEGHEM A.C., LIU J. et coll.: TESE-ICSI: higher fertili-zation and pregnancy rate after intracytoplasmic sperm injection [ICSI] with spermatozoa obtained by testicular biopsy [TESE]. Hum. Re-prod,1995;10: 148-52.

28. SOFFER Y., LEWIN L.M. : Analyse de la structure chromatinienne des spermatozoïdes humains par la cytométrie en flux à l'acridine-orange. Andrologie, 1999; 2:00-00, sous presse.

29. TOURNAYE H. CAMUS M. Goossens et coll.:[1995] Recent concepts in the management of infertility because of non-obstructive azoospermia. Hum Reprod 10, Suppl 1, 115-119.

30. TOURNAYE H., LIU J., NAGY Z. et coll. :The use of testicular sperm for intra-cytoplasmic sperm injection in patients with necrospermia. Fer-til.Steril.,1996; 2: $331-4$.

31. VAN STEIRTEGHEM A. C., NAGY Z., JORIS H. et coll: High fertilization and im-plantation rates after intracytoplasmic sperm insemination. Hum Reprod.,1993; 8: 1061-6.

32. VAN STEIRTEGHEM A, NAGY P, JORIS H, et coll.: Results of intracytoplasmic sperm injection with ejaculated, fresh and frozen-thawed epididymal and testicular spermatozoa. Hum Reprod, 1998;13 [Suppl 1]:134-42.

33. VANDERVORST M, TOURNAYE H., CAMUS M. et coll.: Patients with absolutely immotile spermatozoa and intracytoplasmic sperm injection. Human Re-production , 1997; 12 [11] :2429-33

34. VERHEYEN G, NAGY Z, JORIS H, DE CROO I, TOURNAYE $H$, VAN STEIRTEGHEM A : Quality of frozen-thawed testicular sperm and its preclinical use for intracytoplasmic sperm injection into in vitromatured germinal-vesicle stage oocytes. Fertil Steril, ,1997; 67: 74-80

35. VON ZUMBUSH A, FIEDLER K, MAYERHOFER A, et coll. Birth of healthy chil-dren after intracytoplasmic sperm injection in two couples with male kartagener's syndrome. Fertil. Steril, 1998; 70:643-6

36. WHO Laboratory Manual for the examination of human semen and sperm-cervical mucus interaction. Cambridge University Press,3ème Edi-tion, 1992.

37. WOLF J.PH., FENEUX D., ESCALIER D. et al.: Pregnancy after subzonal insemi-nation with spermatozoa lacking outer dynein arms. J. Reprod.Fertil.,1993; 97, 487-92.

38. ZAMBONI L. [1987] The ultrastructural pathology of the spermatozoon as a cause of infertility: the role of electron microscopy in the evaluation of semen quality. Fertil. Steril., 48, 711-34.

\section{ABSTRACT}

Immobile Fresh or Frozen-thawed Spermatozoa in Assisted Fertilization.

Y. SOFFER1, D. STRASSBURGER2, S. FRIEDLER2, S. KAUFMAN1, A. UMANSKI1 , A. RAZIEL2*, R. RON-EL 2

Contrasting with sperm count or morphology, complete lack of mobile sperm may seriously impair ICSI fertilization and pregnancy rate. In three cases with flagellar skeleton abnormalities [dynein arm absence] only immobile sperm were found in the ejaculate. Following repeated ejaculations, higher rates of viable spermatozoa and even some motile spermatozoa could be found. Some times, in nonobstructive azoospermia, extensive sperm search didn't allow us to find but immobile sperm mostly, with very few motile sperm cells, not enough for the microinjection of all oocytes. The third group of immobile sperm is iatrogenic, following freezing and thawing surgically retrieved, testicular or epididymal spermatozoa in order to avoid repeated surgical retrieval. Following thawing, one find frequently very few motile spermatozoa that may be not enough for all retrieved oocytes and it might be necessary to inject some eggs with immobile spermatozoa. The outcome of ICSI using mobile and immobile sperm was compared in the three above mentioned groups: 1 - immobile ejaculated sperm with flagellar defects, 2immobile sperm discovered in the ejaculate after extensive sperm search and 3-immobile frozen-thawed testicular or epididymal spermatozoa. The results of ICSI in these groups show that fertilizing ability of fresh or frozenthawed immobile spermatozoa is not significantly different from ICSI with mobile sperm from the same origin. More over, in the first group with flagellar abnormalities, repeated ejaculations allowed us significantly increase sperm viability and fertilization ability. Finding only immobile fresh or frozen-thawed sperm the day of egg retrieval should not lead 
us to ICSI cancellation. Pregnancies may occur with such immobile sperm.

Key-words: Immobile sperm; necrozoospermia; flagellar abnormalities; Azoospermia; extensive Sperm search; sperm surgical retrieval; sperm cryopreservation and thawing; assisted fertilization [ICSI]; fertilization rate; pregnancy rate. 